\title{
El rol del profesor y la actividad demostrativa: Una experiencia de aula empleando Geogebra ${ }^{1}$
}

\author{
The teacher's role and demonstration activity: A \\ classroom experience using Geogebra
}

\section{O papel do professor e da atividade demonstração: Uma experiência em sala de aula usando o Geogebra}

Recibido: mayo de 2013

Aceptado: agosto de 2013
Cristina Mejía ${ }^{2}$

Oscar Molina ${ }^{3}$

\section{Resumen}

En esta experiencia de aula presento un ciclo didáctico que forma parte del trabajo de grado que estoy desarrollando para optar por el título de maestría en Docencia de las Matemáticas. Pretendo exponer una experiencia en la que se analiza el rol del profesor, bajo la teoría de la Mediación Semiótica y fundamentado en la aproximación metodológica para la enseñanza del grupo $Æ \cdot G$. Tal ciclo planteado en un aula de octavo grado, favorece la construcción de un sistema teórico local y se emplea un software de geometría dinámica como artefacto.

Palabras clave: Profesor; el papel del profesor; aula; recursos didácticos; recursos informáticos; software; aprendizaje; procesos cognitivos; procesos de justificación; demostración.

\begin{abstract}
In this present classroom experience teaching a course that is part of the thesis I am developing to qualify for the master's degree in Teaching of Mathematics. I intend to present an experience in which he examines the role of the teacher, on the theory of semiotic mediation and based on the methodological approach for teaching $\bullet$ Æ group G. Such a cycle raised in eighth grade classroom, favors the construction of a local theoretical system employing a dynamic geometry software as an artifact
\end{abstract}

Keywords: teacher, the teacher's role; classroom teaching resources, computing resources, software, learning cognitive processes, processes of justification; demonstration.

\section{Resumo}

$\mathrm{Na}$ presente experiência em sala de aula ensinando um curso que faz parte da tese que estou desenvolvendo para se qualificar para o mestrado em

1 Artículo de Investigación.

2 Universidad Pedagógica Nacional. Bogotá, Colombia. Contacto: mdma_cmejia758@pedagogica.edu.co

3 Universidad Pedagógica Nacional. Bogotá, Colombia. Contacto: ojmolina@pedagogica.edu.co 
Ensino de Matemática. Tenho a intenção de apresentar uma experiência na qual ele analisa o papel do professor, na teoria da mediação semiótica e com base na abordagem metodológica para o ensino • Æ grupo G. Tal ciclo levantado em sala de aula da oitava série, favorece a construção de um sistema teórico local, empregando um software de geometria dinâmica como um artefato.

Palavras chave: professor, o papel do professor, recursos pedagógicos em sala de aula, os recursos de computação, software, processos cognitivos de aprendizagem, os processos de justificação; demonstração.

\section{Contextualización:}

Para optar por el título de Maestría en la Universidad Pedagógica Nacional, estoy desarrollando un trabajo tendiente a analizar el rol del profesor bajo la Teoría de la Mediación Semiótica TMS. Para dicho análisis, se llevó a cabo esta experiencia de aula empleando un software de geometría dinámica y un ciclo didáctico inspirado en la aproximación metodológica para la enseñanza del grupo Æ•G de la Universidad Pedagógica Nacional, para favorecer la actividad demostrativa ${ }^{2}$.

Esta experiencia de aula fue llevada a cabo con un grupo de 13 estudiantes de octavo grado de The English School, en el marco de la clase de Geometría. Dicha clase de geometría, no cuenta con un espacio aparte en el horario, se desarrolla como parte del currículo propuesto para matemáticas. Se cuenta con una sala de computadores y un tablero electrónico con videobeam en el salón de clase. The English School es un colegio del Bachillerato Internacional, que tiene su propio modelo curricular, pero también debe atender a los requerimientos del MEN colombiano, por tanto la demostración está incluida en dicho currículo.

\section{Referentes Teórico Prácticos:}

$\mathrm{Al}$ ser el rol del profesor, objeto del análisis de mi trabajo de grado, la experiencia de Aula que presento en este escrito, está basada en el marco teórico de la TMS, que reconoce el potencial semiótico de los artefactos como los software de geometría dinámica, y sostiene que dichos artefactos permiten al profesor, mediante la interacción, emplear los artefactos como herramientas de mediación semiótica (Bartolini-Bussi \& Mariotti, 2008) (Drijvers, Kieran \& Mariotti, 2010). La TMS propone diseñar un ciclo didáctico que favorezca el aprendizaje de los estudiantes. En esta experiencia de aula, el ciclo propuesto, está fundamentado en la aproximación metodológica para la enseñanza del grupo $Æ \bullet G$ de la Universidad Pedagógica Nacional. Espero poner a consideración esta experiencia de aula, en la que reconociendo el potencial semiótico del software de geometría dinámica GeoGebra4, se diseñó e implementó una secuencia didáctica a partir de la cual se pretende analizar del rol del profesor, bajo la TMS.

\section{Descripción General de la Experiencia de Aula:}

La experiencia de aula consistió en tareas propuestas durante aproximadamente 20 sesiones de clase de geometría (un trimestre académico), se inició con una presentación discutida sobre los elementos de visualización y bajo la premisa de que "no todo lo que vemos es, y no todo es como se ve".

Las tres actividades introductorias fueron:

1. Presentaciones VISUALIZACIÓN. A partir de estas presentaciones, se establecieron algunos de los elementos teóricos (definiciones, objetos de la geometría) del sistema teórico local que se construirá con la comunidad de clase. Se estableció el acuerdo de llevar un cuaderno de campo, en el que irían anotando todos los datos relevantes de la clase (Hechos

2 Actividad conformada por dos procesos, el de conjeturación (cuyo producto es una conjetura) y el de justifcación (cuyo producto es la explicación, prueba o demostración del enunciado conjeturado) 
geométricos, definiciones, conclusiones, percepciones, etc.)

2. Actividad de exploración en Geogebra. En la que los estudiantes explorarían una construcción previamente realizada.

3. Construcción en Geogebra. De la figura con la que se realizó la exploración.

Luego de estas actividades introductorias, se les propuso a los estudiantes un conjunto de tareas. Cada tarea propuesta a los estudiantes de octavo grado, pretendía agregar un elemento más al sistema teórico local que se está construyendo con la comunidad de clase, y con ellas se espera que los estudiantes realicen actividad demostrativa a partir de la exploración con el artefacto y el trabajo en grupo.

En este proceso, la profesora propicia de manera intencionada que los estudiantes conecten sus signos y significados personales con signos (que ella considera matemáticos). Para tal efecto, en la clase la profesora genera discusiones instruccionales a partir de los signos producidos -con pequeños grupos de 3 o 4 estudiantes en algunos momentos, y con todo el grupo de 13 estudiantes en otros momentos-, orientando el trabajo, hacia una discusión que los haga evolucionar a signos y significados matemáticos.

El sistema teórico local que se construyó con la comunidad de clase a partir de las tareas propuestas fue el siguiente:

Figura 1.

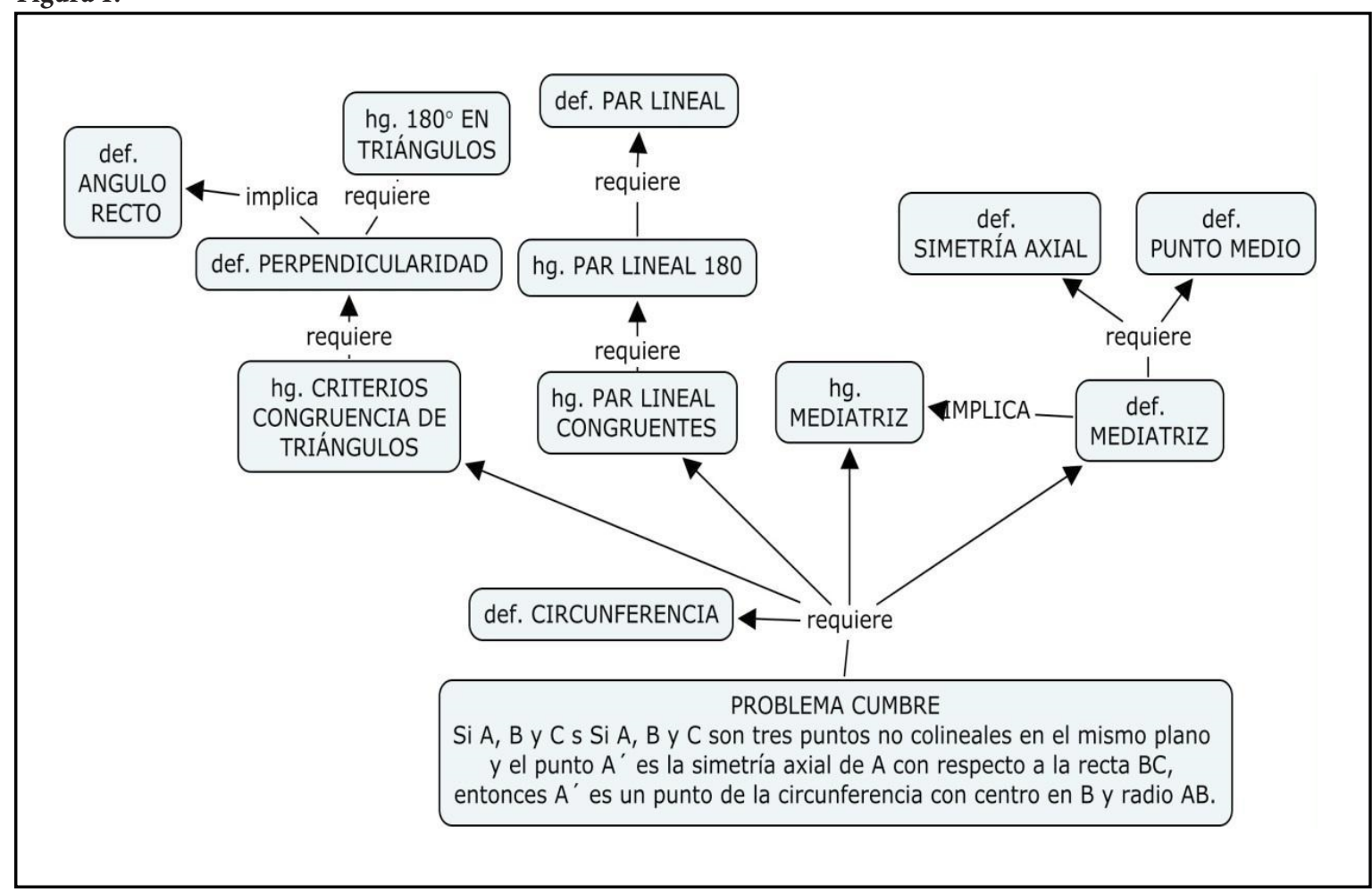

Funete: Elaboración Propia.

\section{Logros y dificultades evidenciadas}

Fue muy placentero ver como los estudiantes se involucraban, en algunos momentos más que en otros, en las discusiones de clase y cómo el ambiente en la clase de geometría evolucionó de tal manera que las discusiones se tornaban más matemáticas y más profundas, el manejo del lenguaje y del artefacto mismo también fue un factor que evolucionó.

También fue importante para mí, ver cómo mi rol como profesora fue significativo al lograr que los signos que emergían, evolucionaran hacia signos matemáticos. 
Por otro lado, fue difícil trabajar con el factor tiempo, y al ser esta una experiencia innovadora en el lugar donde trabajo, los espacios y tiempos fueron muy limitados, seguramente con más tiempo y con un trabajo menos aislado (más profesores involucrados) los resultados habrían sido mucho mejores.

Aunque la actividad demostrativa sí se favoreció, no se logró justificar el problema cumbre por que el semestre escolar terminó.

\section{Reflexión final}

Los avances en mi estudio, me han mostrado que la TMS sí es afortunada para proporcionar una manera de analizar la producción de los estudiantes en un entorno mediado por artefactos y por un experto. También introduce elementos que aunque no son nuevos, sí señalan una nueva vía para enfocar los trabajos de investigación y a su vez una nueva forma de plantear situaciones en las clases, en las que el uso de los artefactos sea intencionado y no pierda de vista las metas educativas.

Por otro lado, la aproximación metodológica del grupo de investigación $Æ \cdot G$ efectivamente favorece la actividad demostrativa por cuanto las tareas propuestas efectivamente redundan en proporcionar evidencias de los procesos de conjeturación y justificación por parte de los estudiantes.

\section{Referencias}

Bartolini Bussi, M.G. \& Mariotti, M.A. (2008). Semiotic mediation in the mathematics classroom: Artifacts and signs after a Vygotskian perspective. En L. English, M.G. Bartolini Bussi, G.A. Jones, R.A. Lesh y B. Sriraman (Eds.), Handbook of interna- tional research in mathematics education (segunda edición revisada, pp. 746-783). Mahwah, EUA: Lawrence Erlbaum.

Drijvers, P., Kieran, C. y Mariotti, M.A. (2010). Integrating technology into mathematics education: Theoretical perspectives. En C. Hoyles y J.-B. Lagrange (Eds.), Mathe- matics education and technology - Rethinking the terrain. The 17th ICMI Study (pp. 89-132). New York, EUA: Springer.

Mariotti, M.A. (2009). Artifacts and signs after a Vygotskian perspective: The role of the teacher. ZDM Mathematics Education, 41(4), 427-440.

Samper, C., Perry, P., Camargo, L. y Molina, Ó. (Sometido a consideración). Innovación en un aula de geometría de nivel universitario, Universidad Pedagógica Nacional, Bogotá, Colombia. 